\title{
A Decision Support System for Planning Promotion Time Slots *
}

\author{
P. A. Pereira ${ }^{1}$, F. A. C. C. Fontes ${ }^{1}$, and D. B. M. M. Fontes ${ }^{2}$
}

1 Dept. of Mathematics for Science and Technology, Universidade do Minho, 4800-058 Guimarães, Portugal. ffontes; ppereira@mct.uminho.pt

${ }^{2}$ Faculdade de Economia da Universidade do Porto, Rua Dr. Roberto Frias, 4200-464 Porto, Portugal. fontes@fep.up.pt

\begin{abstract}
We report on the development of a Decision Support System (DSS) to plan the best assignment for the weekly promotion space of a TV station. Each product to promote has a given target audience that is best reached at specific time periods during the week. The DSS aims to maximize the total viewing for each product within its target audience while fulfilling a set of constraints defined by the user. The purpose of this paper is to describe the development and successful implementation of a heuristic-based scheduling software system that has been developed for a major Portuguese TV station.
\end{abstract}

Key words: Decision support systems, scheduling, heuristics.

\section{Introduction}

Decision Support Systems (DSS) are used to support decision making in almost every area of business. In this work we report on a DSS specifically developed for a Portuguese TV station. As noted by Kendall and Kendall ([7], pp. 320-329), all DSS methodology can fall into the two categories of analytic or heuristic. Analytic DSS that use optimization procedures have been used to support the scheduling of personnel, equipment and even value chain activities [8]. Unfortunately, the size and time requirements to generate even small assignment schedules

\footnotetext{
* Research supported by SIC and FCT/POCI 2010/FEDER through Project POCTI/MAT/61842/2004.
} 
using an analytic method can be prohibitive. Hence, a heuristic DSS assignment schedule is proposed.

Optimization techniques have been successfully employed in various fields, however only a few studies exist that address the optimization problems in the television and media industry. The majority of these studies focus on scheduling television programs and on modelling audience and audience behaviour rather than scheduling breaks. The literature dealing specifically with TV breaks scheduling problem is sparse and deals only with commercial breaks. Bollapragada et al. [2, 3] studied the commercial scheduling problem to generate sales plans to meet the requirement of a single advertiser. The problem was modelled as an integer program and solved sequentially for each advertiser with an objective to make the least use of premium inventory. Bollapragada [4] then studied the problem on scheduling commercials over a specific period so that the airing of the same commercials are spread as evenly as possible. Jones [6] introduced the advertising allocation problem as an example to design incompletely specified combinatorial auctions where potentially hundreds of advertisers can submit combinatorial bids for the airing of their commercials in the advertising slots. The problem was modelled as an integer program and heuristics based on constraint programming were used to find feasible solutions. Based on this work, Zhang [9] proposed a two-step hierarchical approach. First, a winner determination problem is solved to select advertisers and assign them to shows. Then, a pod assignment problem is used to schedule selected advertisers commercials to slots within a specific show. In another recent work Mihiotis and Tsakiris [5] have studied the advertising allocation problem but from the advertising company point of view. More specifically, they solve the problem of deciding where the commercials are to be place, given the set of available places, their costs, and the number of people viewing each one of them. The choices are to be made in order to have the maximum total of people viewing subject to a budget constraint. They developed a binary mathematical programming model that, due to the enormous number of variables, is then solved heuristically.

The problem considered in this paper takes as input a list of breaks, a list of show spots, and a list of requirements for shows that the marketing department would like to satisfy. The objective is to build an assignment schedule that maximizes the total viewing for each product within its target audience satisfying several constraints and fulfilling the audience targets. We name this problem TV Self-Promotion Assignment Scheduling Problem (TSPASP). 


\section{Problem Description and Formulation}

In a TV channel week there are several self promotion breaks, that is, slots of time in between shows that are not allocated to commercial purposes. These breaks, from now on referred to simply as breaks, are to be used to advertise shows that are still to be broadcasted and also own merchandizing. There are a number of advertising campaigns, that we call spots, from which we must choose the ones to be used in the aforementioned breaks.

The TSPASP described below essentially consists in assigning a subset of the existing spots, each of which can be used more that once, to the available breaks, subject to audience, operating and legal constraints. Each break is characterized by the broadcasting time, its duration, and its forecasted audience in each segment, called target. Each existing advertising spot is characterized by the show/product (for the sake of exposition form now on we will use show only) they advertise, by its duration, and by the nature o the advertised show (i.e a spot referring to show that shows alcohol or sex may only be broadcasted after 22:30). However, there are other issues that must be accounted for. For instance, a show must not be advertised after it has been broadcasted. Associated with each show there are requirement that must be satisfied, such as number of times a show must be advertised, how show advertisements should be spread over the week, the number of people that has seen at least one spot for the show, and the number of times that a show has been advertised to its intended audience.

Let $c_{i t}$ be the number of contacts forecasted at break $i$ for target $t$ and let $p_{j k}$ and $q_{i j}$ be binary parameters denoting whether spot $j$ is of product $k$ and spot $j$ is intended for target $t$. Since we must allocate spots to breaks, we define binary variables $x_{i j}$ that are to be set to 1 if at break $i$ spot $j$ is broadcasted and set to 0 otherwise. The mathematical programming model $(\mathcal{P})$, allows to determine the spot broadcast decisions, which are made in order to maximize total viewing for each product within its target audience, as given by equation (1), and must satisfy the constraints given in equations (2) to (9).

The first 4 constraints, equations (2) to (5), are show constraints and establish that each show must have a minimum percentages $S_{k_{m i n}}$ of broadcasted spots within pre-specified time intervals $I_{l}$ (i.e. up to 2 hours before the show being broadcasted and in each day $I_{d}$, until it is broadcasted; has advertising maximum and minimum limits; and must have a pre-specified minimum cover $C_{k t_{m i n}}$ in its targeted audience. The following 2 constraints specify maximum and minimum limits for the number of times that each spot is broadcasted and also intervals of time 
$F_{i}$ when spot broadcasting is forbidden, either due to legal constraints or to operational ones. In equation (8) we imposed that the duration $s_{j}$ of the spots broadcasted in each break does not exceed break duration $b_{i}$. Finally, the binary nature of the decisions to be made is given by equation (9).

$$
\begin{array}{rlr}
(\mathcal{P}) \text { Maximize } & G R P^{\prime} s=\sum_{i} \sum_{j} \sum_{k} c_{i t} \cdot x_{i j} \cdot q_{j t} \\
\text { subject to } & & \\
& S_{k_{\text {min }}} \sum_{i} \sum_{j} x_{i j} \cdot p_{j k} \leq \sum_{i \in I_{l}} \sum_{j} x_{i j} \cdot p_{j k} & \forall k, I_{l} . \quad(2) \\
& D_{\min } \sum_{i} \sum_{j} x_{i j} \cdot p_{j k} \leq \sum_{i \in I_{d}} \sum_{j} x_{i j} \cdot p_{j k} & \forall k, I_{d} \cdot(3) \\
& K_{\min } \leq \sum_{i} \sum_{j} x_{i j} \cdot p_{j k} \leq K_{\max } & \forall k . \quad(4) \\
& \sum_{i} \sum_{j} x_{i j} \cdot p_{i k} \cdot c_{i t} \geq C_{k t_{\text {min }}} & \forall k, t . \quad(5) \\
& S_{\text {min }} \leq \sum_{i} x_{i j} \cdot p_{j k} \leq S_{\max } & \forall j . \quad(6) \\
& \sum_{j} x_{i j}=0 & \forall j \in F_{i}(7) \\
& \sum_{j} s_{j} \cdot x_{i j} \leq b_{i} & \forall i . \quad(8) \\
& x_{i j} \in\{0,1\} 0 & \forall i, j . \quad(9)
\end{array}
$$

\section{Methodology}

The methodology proposed is a decision support system, that we have named PlanOptimUM, which includes a heuristic procedure to generate solutions. After discarding the non feasible solutions, the remainder are evaluated. The best solutions are then suggested to the operator that through editing can include some extra elements, not provided to the PlanOptimUM. These changed solutions can then be re-evaluated in order to choose the most convenient one.

Solutions Generation: In order to generate solutions we have implemented an heuristic procedure that outputs spot-break assignment binary matrices. The solution procedure has basically 4 stages: In stage (i) the show-defined constraints are converted into spot-constraints. Therefore, except for the break duration constraints which are dealt with differently, we only have spot-constraints. Although some constraints are easily converted, others have required complex procedure to do so. This is the case of the minimum coverage constraints. We have developed an estimator for the number of times each show would need to be advertised since the coverage is a nonlinear and unknown function of the broadcasted spots. In stage (ii) we compute the maximum $M b_{j}$ and the minimum $m b_{j}$ number of times that spot $j$ may be used. In stage (iii) we generate the binary matrix by resorting to an iterative greedy heuristic procedure based on the following 
- select the spot having the largest value of the remaining times to be used. (Initially $m b_{j}$.)

- from the breaks that still have free time, select the break having the highest audience rate for the target of selected spot.

In stage (iv) even if the solution is feasible (which typically happens) we look for breaks with available time in order to improve the solution. For each break we iteratively select a spot/show for which the target has the highest audience rating whose duration is compatible with the remaining break time.

User Interface: The software system used in the development of the computer programs that comprise the PlanOptimUM was MATLAB and $\mathrm{C}$ for model solving and Visual Basic for the interface.

Given the complexity of the problem many of the objectives that management wanted to maximize and minimize have been converted into constraints. In seeking a maximum amount of autonomy in the use of this system the interface developed allows for constraint introduction. Furthermore, the PlanOptimUM, through the use of the interface can easily be used to experiment other schedules and scenarios without consuming much time.

PlanOptimUM generates the spot-break assignment schedules. The schedules can then be edited by an operator, that can directly alter in order to incorporate his/her own personal and subjective judgment into the assignment process. Editing and customization features of the system included the ability to override the schedule and make preassignments of spots to breaks.

In Figure 1 we show how the interface looks like, when the operator is changing show characteristics and editing the solution.

\section{Conclusions}

The application of the PlanOptimUM took place at the SIC-Sociedade Independente de Comunicao SA, in Carnaxide, Portugal. SIC is an overthe-air commercial television that frequently leads audience shares in Portugal. The management of SIC intended to use the PlanOptimUM to prepare weekly schedules for spot assignments to the breaks.

On average there are about 50 shows and products to be advertised each week and the number of different spots to advertise each one of them varies between 1 to 5 . On one week there are about 230 self promotion breaks to which spots must be assigned to. SIC management wanted a reduction in the time required to establish weekly spot assignment schedules. This has been greatly achieved since the work of more 

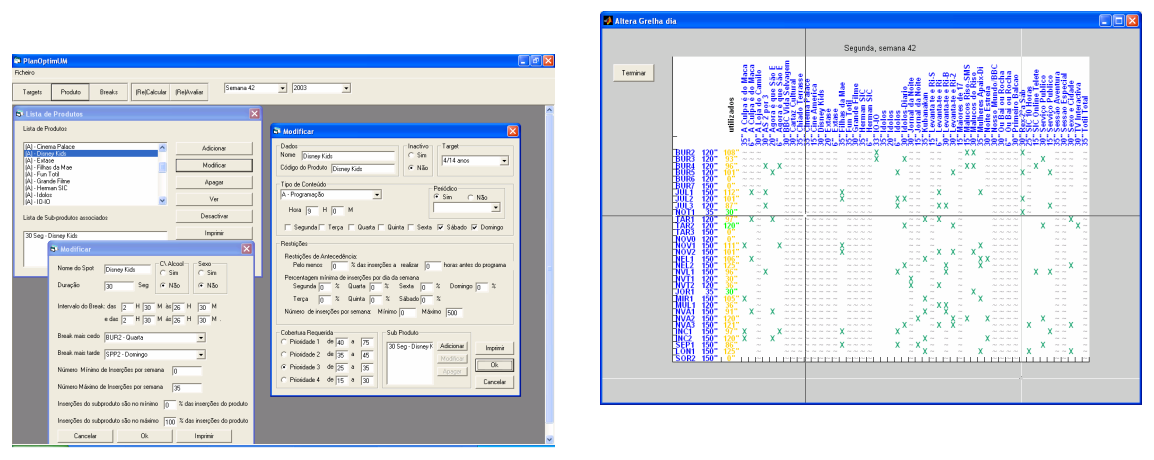

Fig. 1. Solution tuning.

than one person during a week is now done by the one person in one afternoon. A comparison of three months observations on both manually generated schedules and the PlanOptimUM generated schedules has shown a substantial qualitative improvement in scheduling.

A natural improvement, which is already being explored, is to include in this system optimization scheduling techniques.

\section{References}

1. Benoist T., Bourreau E., Rottembourg B.: The TV-Break Packing Problem, European Journal of Operational Research 176 (3):1371-1386, 2007.

2. Bollapragada S., Cheng H., Philips M., Garbiras M., Scholes M., Gibbs T., and Humhreville M.: NBCs Optimization Systems Increase Revenues and Productivity, Interfaces 32 (1): 47-60, 2002.

3. Bollapragada S., Garbiras M.: Scheduling commercials on broadcast television, Operations Research 52 (3): 337-345, 2004.

4. Bollapragada S., Bussieck M.R., Mallik S.: Scheduling commercials videotapes in broadcast television, Operations Research 52 (5): 679-689, 2004.

5. Mihiotis A., Tsakiris I.: A mathematical programming study of advertising allocation problem, Applied Mathematics and Computation 148 (2): 373-379, 2004.

6. Jones J. J., Tsakiris I.: Incompletely Specified Combinatorial Auction: An Alternative Allocation Mechanism for Business to Business Negotiations, Ph.D. Dissertation, University of Florida, FL, 2000.

7. Kendall K.E. and Kendall J.E.: Systems Analysis and Design, (Prentice Hall, Englewood Cliffs, NJ, 1988.

8. Shapiro J.F., Singhal V.M. and Wagner S.N.: Optimizing the Value Chain, Interfaces 23 (2): 102- 117, 1993.

9. Zang Y: Mathematical models for the television advertising allocation problem, International Journal of Operational Research 1, 302-322, 2006. 